\title{
PEIXES PEIÁGICOS DA LAGOA RODRIGO DE FREITAS, RIO DE JANEIRO, BRASIL
}

\author{
Pelagics fishes of Rodrigo de Freitas Lagoon, \\ Rio de Janeiro, Brazil
}

\author{
José V. Andreata ${ }^{1}$ \\ Luiz O. V. Oliveira ${ }^{1}$ \\ Bruno C. Meurer ${ }^{1}$ \\ Natalie V. Freret ${ }^{1}$ \\ Dirceu E. Teixeira ${ }^{1}$ \\ Felipe V. Manzano ${ }^{1}$ \\ Michele M. Longo ${ }^{1}$
}

\section{Resumo}

O objetivo desse estudo é caracterizar a composição da ictiofauna pelágica, a distribuição espacial/sazonal e a abundância relativa na Lagoa Rodrigo de Freitas. As coletas foram realizadas de março de 1991 a abril de 2004 em quatro áreas, com o auxílio de tarrafas, arrasto-de-praia, puçá e rede-de-espera. No laboratónio, o material foi identificado, pesado e medido. Foram realizadas 103 coletas, sendo capturados 113.109 espécimes, correspondendo a 65 espécies, das quais 104.790 espécimes foram pelágicas, correspondendo a 35 espécies. A abundância relativa das 8 espécies mais representativas mostrou Poecilia vivipara com 20,86\%; Phalloptychus januarius com 20,71\%; Atherinella brasiliensis com 17,56\%; Jenynsia multidentata com 15,21\%; Brevoortia aurea com 10,31\%; Brevoortia pectinata com 6,65\%; Mugil sp. com 6,11\% e Mugil liza com $1,00 \%$, enquanto que as demais espécies representaram $1,59 \%$ do total capturado. A abundância relativa total por áreas foi de 19,79\% para a área 1, 29,96\% para a área 2, 23,90\% para a área 3 e 26,35\% para a área 4.

Palavras-chave: Composição; Peixes pelágicos; Lagoa.

1 Laboratório de Ictiologia, Instituo de Ciências Biológicas e Ambientais, Universidade Santa Úrsula. Rua Fernando Ferrari, 75, Botafogo, Rio de Janeiro, 22.231-040 - luizotavio01@yahoo.com 


\section{Abstract}

The propouse of this work is to characterize the composition, the spatial/seazonal distribution and the relative abundance of pelagic ichthyofauna over Rodrigo de Freitas Lagoon. Samples were accomplished during March 1991 and April 2004 in four areas using hand net, beach seines cast net and gill net. All collected material was identified, weighted and messured at the laboratory. It was accomplished 103 samples and 113109 specimens were captured, corresponding to 65 species, of which 104790 specimens are pelagics, corresponding to 35 species. The relative abundace of pelagic ichthyofauna showed that $20.86 \%$ are Poecilia vivipara; $20.71 \%$ are Phalloptychus januarius; $17.56 \%$ are Atherinella brasiliensis; $15.21 \%$ are Jenynsia multidentata; $10.31 \%$ are Brevoortia aurea; $6.65 \%$ are Brevoortia pectinata; $6.11 \%$ are Mugil sp.; $1.00 \%$ are Mugil liza while the other species represented $1.59 \%$ from total captured. The total relative abundance showed that area 1 had 19.79\%, area 2 had 29.96\%, area 3 had $23.90 \%$ and area 4 had 26.35\%.

Keywords: Composition; Pelagic fishes; Lagoon.

\section{Introdução}

A Lagoa Rodrigo de Freitas, dentre as demais lagoas costeiras do Estado do Rio de Janeiro, tem despertado a atenção de cientistas, urbanistas e da população estabelecida no entorno da lagoa, devido aos acentuados fenômenos naturais que ocorrem, como: estagnação e deterioração da qualidade da água, exalação de gases, assoreamento e grandes mortandades de peixes. Uma lagoa por sua natureza é um ecossistema frágil, suscetível às ações naturais, porém nos últimos séculos a Lagoa Rodrigo de Freitas sofreu uma forte ação antrópica, que vem acelerando os processos naturais de degradação ambiental da área, especialmente quanto aos despejos dos efluentes domésticos que alteram significativamente a qualidade de suas águas. Estes fatos, relacionados aos problemas antigos já constatados na Lagoa, resultaram na atual situação que se encontra este ecossistema e conseqüentemente a sua ictiofauna (MARCA; ANDREATA, 2001). A composição ictiofaunística da Lagoa Rodrigo de Freitas é característica, apresentando espécies dulcícolas, marinhas e principalmente aquelas que toleram uma grande variação do gradiente de salinidade.

O objetivo desse estudo é caracterizar a composição da ictiofauna pelágica, a distribuição espacial/sazonal e sua abundância relativa nesse ecossistema.

\section{Descrição da área}

A Lagoa Rodrigo de Freitas encontra-se na zona Sul da cidade do Rio de Janeiro, entre as latitudes 22 $57^{\prime \prime 2} 22^{\prime \prime}$ / 22 $2^{\circ} 58^{\prime} 09^{\prime \prime} \mathrm{S}$ e as longitudes

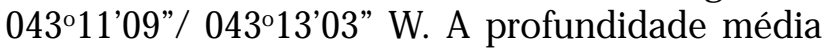
é de 2,81m podendo alcançar no máximo 10,10m (ANDREATA et al., 2002). O sedimento é composto por uma vaza muito fina que forma uma grande coluna vertical, onde se encontram retidos metais pesados e gases tóxicos (KOBLTZ et al., 2001). A ligação com o mar é feita através do canal do Jardim de Alah e sua bacia hidrográfica é formada pelos rios Macacos, Rainha e Cabeça.

A região foi dividida em 4 áreas de coleta de acordo com suas características ambientais. A área 1 situa-se próximo ao Canal do Jardim de Alah, seguindo-se até as proximidades do clube de remo do Flamengo, com vegetação marginal composta por uma estreita faixa de Typha domingensis (taboa), Paspalum vaginatus e de vegetação submersa como Ruppia marítima e Enteromorpha spp. A área 2 está localizada próximo ao Parque da Catacumba, com vegetação de manguezal como Laguncularia racemosa, Rhizophora mangle e alguns exemplares de Avicennia schaueriana e de vegetação submersa como na área anterior. A área 3 situa-se nas proximidades do Clube Piraquê onde recebe águas dos rios Macacos, Rainha, Cabeça e do canal de recirculação (canal do Jóquei). A vegetação é composta por uma faixa estreita de manguezal com exceção de Avicennia schaueriana, acrescidas de Acrostichum aureum, Alternantera philoxeroides e de vegetação submersa como nas áreas anteriores. A área 4 localiza-se na região nordeste da Lagoa, sua vegetação é composta como na área 3 (Figura 1). 


\section{Metodologia de trabalho}

Foram realizadas coletas mensais no período de março de 1991 a abril de 1995 e em meses alternados de abril de 1995 a abril de 2004, nas quatro áreas da Lagoa. Os peixes foram coletados com o auxílio de 4 instrumentos de captura. Para a coleta marginal foi realizado um estudo quantitativo e qualitativo e utilizaram-se: tarrafas com malhas de 15, 18 e $20 \mathrm{~mm}$, com esforço de 30 lances, arrasto-de-praia (picaré), com malhas de 3 e 15 $\mathrm{mm}$, com um esforço de 3 arrastos numa distância de 50 metros e puçá, com malha de $3 \mathrm{~mm}$, com um esforço de 2 lances, numa distância de 50 metros. Uma rede-de-espera, com malhas de 15, 20, 25 e $35 \mathrm{~mm}$, foi deixada na área 1, à deriva durante todo o período da coleta, aproximadamente 4 horas, para o estudo qualitativo e para a abundância geral. Após a captura, os peixes foram acondicionados em sacos plásticos etiquetados com os dados locais e conservados em caixas de isopor contendo gelo e levados para o laboratório, para a triagem. Em seguida, o material foi triado, separando-se os peixes por espécie, área de coleta e instrumento de captura. Cada exemplar foi medido o comprimento-padrão em milímetros, utilizando-se um ictiômetro; o peso total foi aferido em gramas, através de uma balança de precisão.

\section{Resultados e Discussã o}

Durante os treze anos de coleta, foram capturados 113.109 espécimes, dos quais 104.790 foram pelágicos, correspondendo a trinta e cinco espécies (Tabela 1). Das 35 espécies pelágicas, 3 são dulcícolas e 32 são de origem marinha. A abundância relativa das 8 espécies mais representativas mostrou Poecilia vivipara com 20,86\%; Phalloptychus januarius com 20,71\%; Atherinella brasiliensis com 17,56\%; Jenynsia multidentata com 15,21\%; Brevoortia aurea com 10,31\%; Brevoortia pectinata com 6,65\%; Mugil sp. com 6,11\% e Mugil liza com 1,00\% (Figura 2). A análise da sazonalidade total demonstrou um maior percentual de captura na primavera com $32,52 \%$, seguido pelo verão com $25,60 \%$, outono com $23,62 \%$ e do inverno com 18,26\% (Figura 3). A análise da abundância relativa total por área de coleta revelou como mais representativa a área 2 , com 29,96\% do total capturado, seguida da área 4 com 26,35\%, da área 3 com 23,90\% e da área 1 com 12,98\% (Figura 4).

Oliveira (1976) apresentou 41 espécies de peixes para a Lagoa Rodrigo de Freitas, sendo a maioria espécies de origem marinha e de importância comercial, ressaltando a boa condição hidrodinâmica do canal naquela época.

Os baixos valores no gradiente de salinidade evidenciam que a Lagoa não se apresenta como um ambiente composto predominantemente por espécies marinhas, como foi verificado por Andreata et al. (1998, 2002), notando-se atualmente um predomínio de espécies dulcícolas, devido provavelmente à precariedade das condições hidrodinâmicas do canal do Jardim de Alah, e de menor influência, os aportes fluviais.

As lagoas costeiras de Cabiúnas e Comprida na região de Macaé, ecossistemas que apresentam uma comunicação com o mar através da abertura ou fechamento por causas naturais (Reis et al.,1998) apresentou uma menor diversidade de ictiofauna em relação à Lagoa Rodrigo de Freitas. Trabalhos de Aguiaro e Caramaschi (1995) classificaram a lagoa Cabiúna como de água doce, porém $56,67 \%$ dos peixes foram classificados como marinhos, enquanto que $87,5 \%$ dos peixes da lagoa Comprida foram classificados como dulcícolas, sendo apenas uma única ocorrência de exemplar de origem marinha.

Em contrapartida, algumas espécies de peixes como os barrigudinhos (Poecilia vivipara, Phalloptychus januarius e Jenynsia multidentata) passaram a encontrar na Lagoa condições ideais para o crescimento de suas populações. Atualmente estão entre as quatro espécies mais abundantes, e possuem um importante papel ecológico no ecossistema, pois atuam no controle de larvas de insetos transmissores de doenças encontrados na Lagoa como Díptera, Odonata e Hemíptera (Andreata et al., 2001) além de serem utilizados como alimento para diversas espécies de aves e peixes de maior porte.

Nas lagoas de Jacarepaguá e Tijuca, as espécies Poecilia vivipara, Phalloptychus januarius, Atherinella brasiliensis e Jenynsia multidentata (Andreata et al., 1989, 1992) foram classificadas como constantes, com ocorrência acima de 50\%, mostrando uma similaridade com a Lagoa Rodrigo de Freitas. Na Laguna de Marapendi das 10 espécies mais abundantes que perfizeram um total de $95 \%$ do total capturado, apenas três 
(Brevoortia pectinata, Eucinostomus argenteus e Diapterus lineatus) corresponderam as espécies marinhas que penetram no estuário como juvenis utilizando a área para criação (Andreata et al., 1990). Na Laguna da Tijuca foi verificado três regiões distintas dentro da laguna (Andreata et al. (1990a).

A comunidade de peixes pelágicos da Lagoa Rodrigo de Freitas não apresentou um padrão de composição ao longo dos anos. Em relação ao total de espécies pelágicas capturadas,
91,40\% correspondem a 32 espécies marinhas e 8,60\% para 3 espécies dulcícolas. A distribuição sazonal indicou maior percentual de captura na primavera, com $32,52 \%$ e no verão com $25,60 \%$, sendo os menores percentuais no outono, com $23,62 \%$ e inverno com $18,26 \%$ do total capturado. A distribuição por área de coleta mostrou para a área 2 maiores percentuais de captura com 29,96\%,

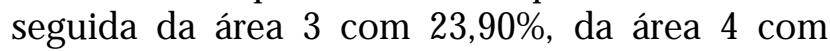
26,35\% e da área 1 com 19,79\%.

\section{Tabela 1 - Lista das espécies pelágicas encontradas na Lagoa Rodrigo de Freitas, por esta ções do a no e por áreas de coleta.}

\begin{tabular}{|c|c|c|c|c|c|c|c|c|}
\hline \multirow{2}{*}{ ESPÉCIES } & \multicolumn{4}{|c|}{ Estação do ano } & \multicolumn{4}{|c|}{ Áreas de coleta } \\
\hline & Primavera & Verão & Outono & Inverno & Área 1 & Área 2 & Área 3 & $\overline{\text { Área } 4}$ \\
\hline Atherinella brasiliensis & 5098 & 5738 & 3303 & 4260 & 3934 & 6861 & 5320 & 2282 \\
\hline Strongylura manina & - & - & - & 1 & 1 & 3 & - & - \\
\hline Caranx latus & 3 & 3 & 26 & 24 & 56 & 5 & 1 & - \\
\hline Oligoplites saurus & 1 & 23 & 1 & 3 & 5 & - & - & - \\
\hline Trachinotus carolinus & 2 & 12 & 2 & 3 & 13 & 2 & 1 & 3 \\
\hline Centropomus parallelus & 50 & 25 & 47 & 13 & 36 & 41 & 32 & 26 \\
\hline Centropomus undecimalis & 42 & 27 & 107 & 87 & 77 & 116 & 31 & 39 \\
\hline Brevoortia pectinata & 2098 & 1262 & 2389 & 1219 & 1474 & 1780 & 1311 & 2403 \\
\hline Elops saurus & 34 & 63 & 109 & 10 & 198 & 4 & 8 & 6 \\
\hline Anchoa januaria & 3 & 33 & 11 & 23 & 23 & 10 & 29 & 8 \\
\hline Mugil liza & 388 & 267 & 301 & 93 & 392 & 141 & 328 & 188 \\
\hline Phalloptychus januarius & 8721 & 4738 & 5951 & 2292 & 4084 & 8166 & 3813 & 5638 \\
\hline Jenynsia multidentata & 6460 & 3966 & 2716 & 2793 & 2223 & 4305 & 4253 & 5154 \\
\hline Poecilia vivipara & 7868 & 5007 & 5700 & 3282 & 5167 & 6512 & 3856 & 6322 \\
\hline Syngnathus rousseau & - & - & 1 & - & 1 & - & - & - \\
\hline Trachinotus falcatus & - & 7 & 2 & - & 3 & 1 & 1 & 4 \\
\hline Mugil curema & 88 & 168 & 171 & 56 & 288 & 67 & 75 & 53 \\
\hline Harengula clupeola & 26 & 4 & 3 & 1 & 31 & 2 & - & 1 \\
\hline Oostethus lineatus & 5 & - & 6 & - & 6 & 2 & 2 & 1 \\
\hline Chaetodipterus faber & - & - & 1 & 3 & 1 & 1 & - & 2 \\
\hline Anchoa tricolor & - & 4 & - & - & 3 & 1 & - & - \\
\hline Poecilia reticulata & 39 & 16 & 11 & 8 & - & 52 & 19 & 3 \\
\hline Lutjanus analis & - & - & 1 & - & 1 & - & - & - \\
\hline Pomatomus saltatrix & 1 & 1 & 3 & 2 & 7 & - & - & - \\
\hline Mugil gaimardianus & - & 19 & 11 & - & 19 & 5 & - & 6 \\
\hline Mugil platanus & 2 & - & 4 & 15 & 3 & 2 & 14 & 2 \\
\hline Anchoviella lepidentostole & - & 18 & 33 & 124 & 65 & 15 & 5 & 90 \\
\hline Xiphophorus helleri & - & - & 1 & - & - & - & 1 & - \\
\hline Brevoortia aurea & 1616 & 5100 & 2836 & 1249 & 1447 & 2276 & 3460 & 3619 \\
\hline Mugil trichodon & - & - & - & 1 & 1 & - & - & - \\
\hline Mugil sp. & 1529 & 310 & 991 & 3570 & 1146 & 1018 & 2477 & 1759 \\
\hline Caranx crysos & 1 & 20 & 9 & - & 30 & - & - & - \\
\hline Chloroscombrus chrysurus & - & - & 1 & - & - & 1 & - & - \\
\hline Sardinella brasiliensis & 3 & - & - & - & 3 & - & - & - \\
\hline Pseudocaranx dentex & - & - & 1 & - & 1 & - & - & - \\
\hline TOTAIS GERAIS & 34078 & 26831 & 24749 & 19132 & 20739 & 31389 & 25037 & 27609 \\
\hline TOTAIS \% & 32,52 & 25,60 & 23,62 & 18,26 & 19,79 & 29,96 & 23,90 & 26,35 \\
\hline
\end{tabular}




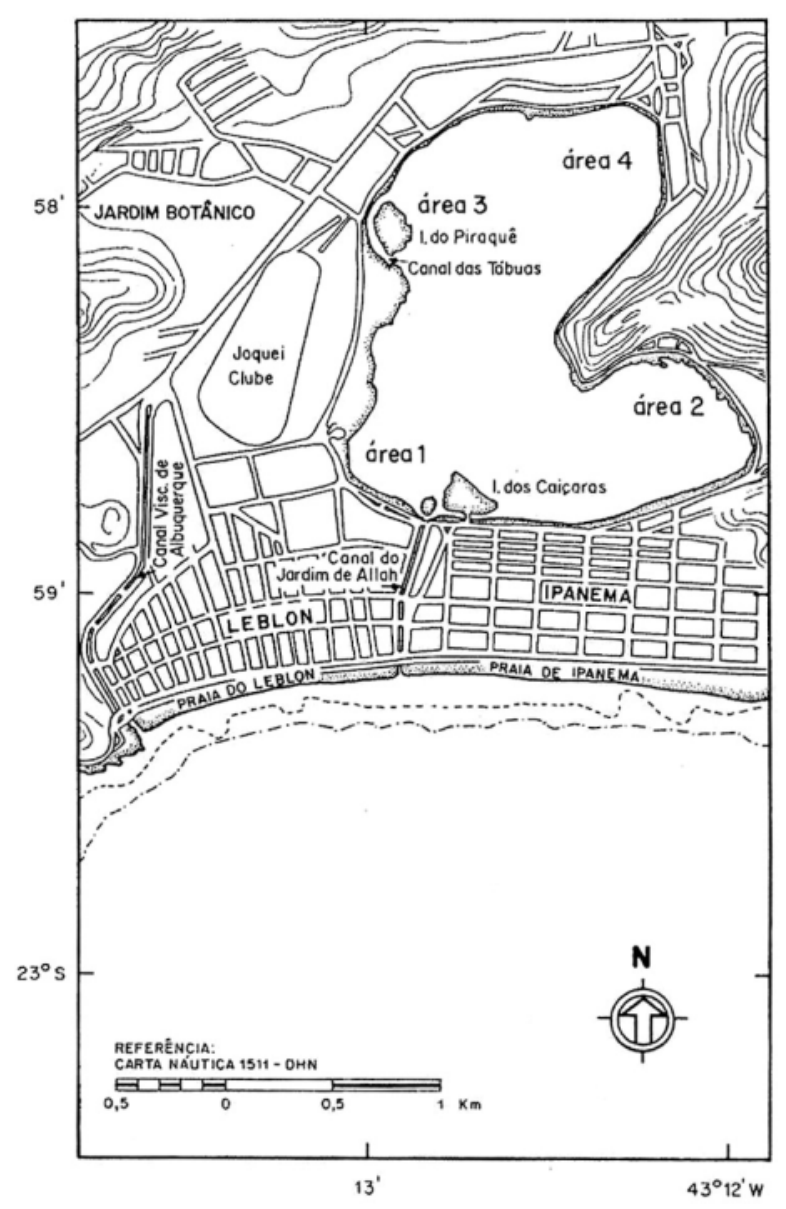

Figura 1 - Áreas de coleta na Lagoa Rodrigo de Freitas, Rio de Janeiro.s

$\Pi=104790$

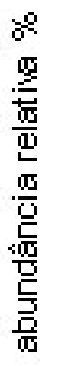
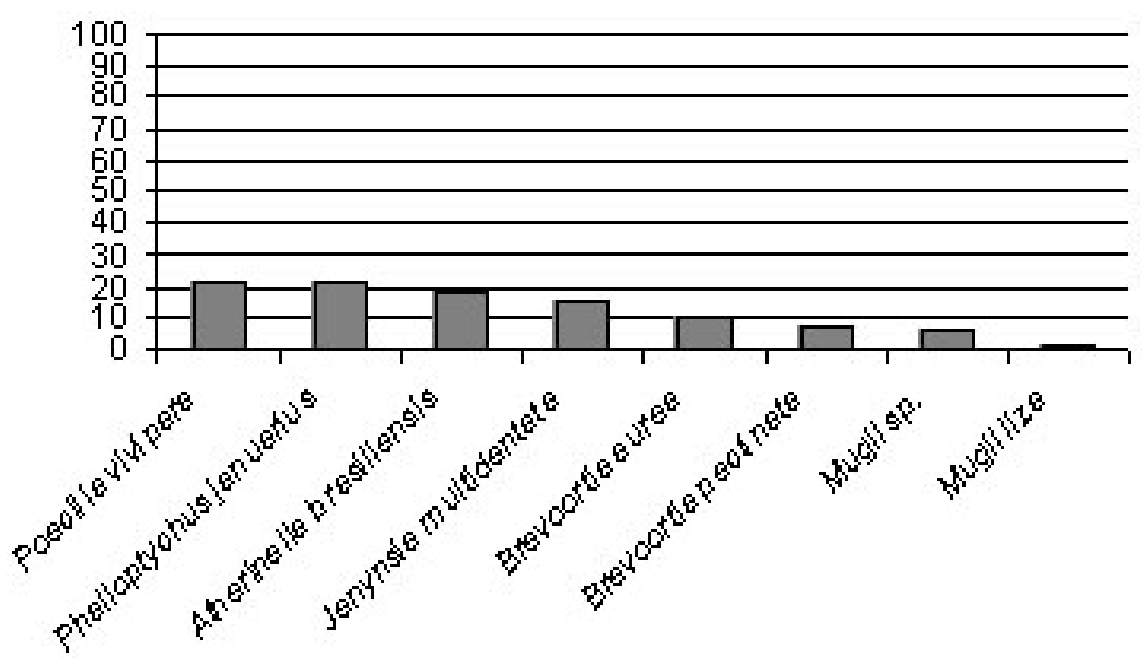

espécies mais representativas

Figura 2 - Abundancia relativa das oito espécies mais representativas na Lagoa Rodrigo de Freitas, Rio de Janeiro. 
rF 104790

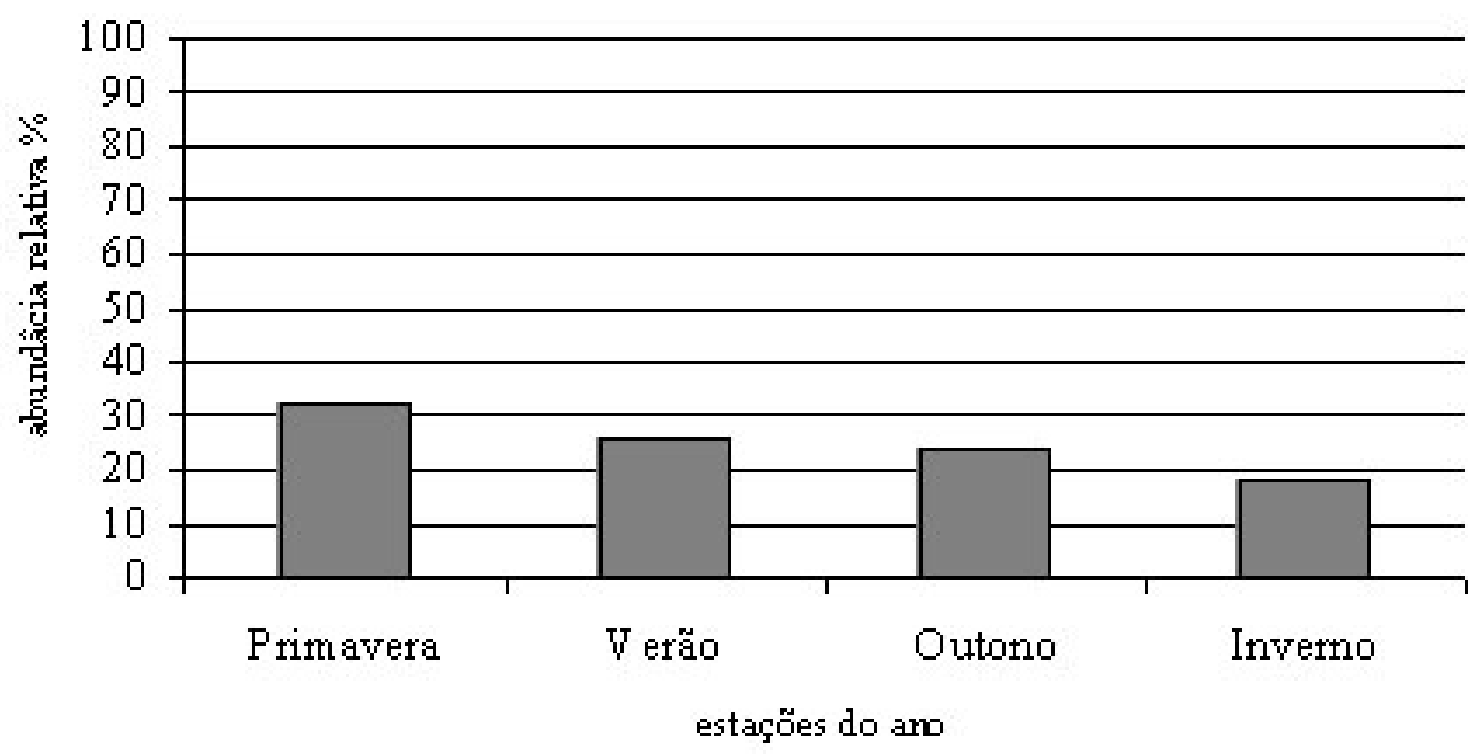

Figura 3 - Percentual de captura por estação do ano na Lagoa Rodrigo de Freitas, Rio de Ja neiro.

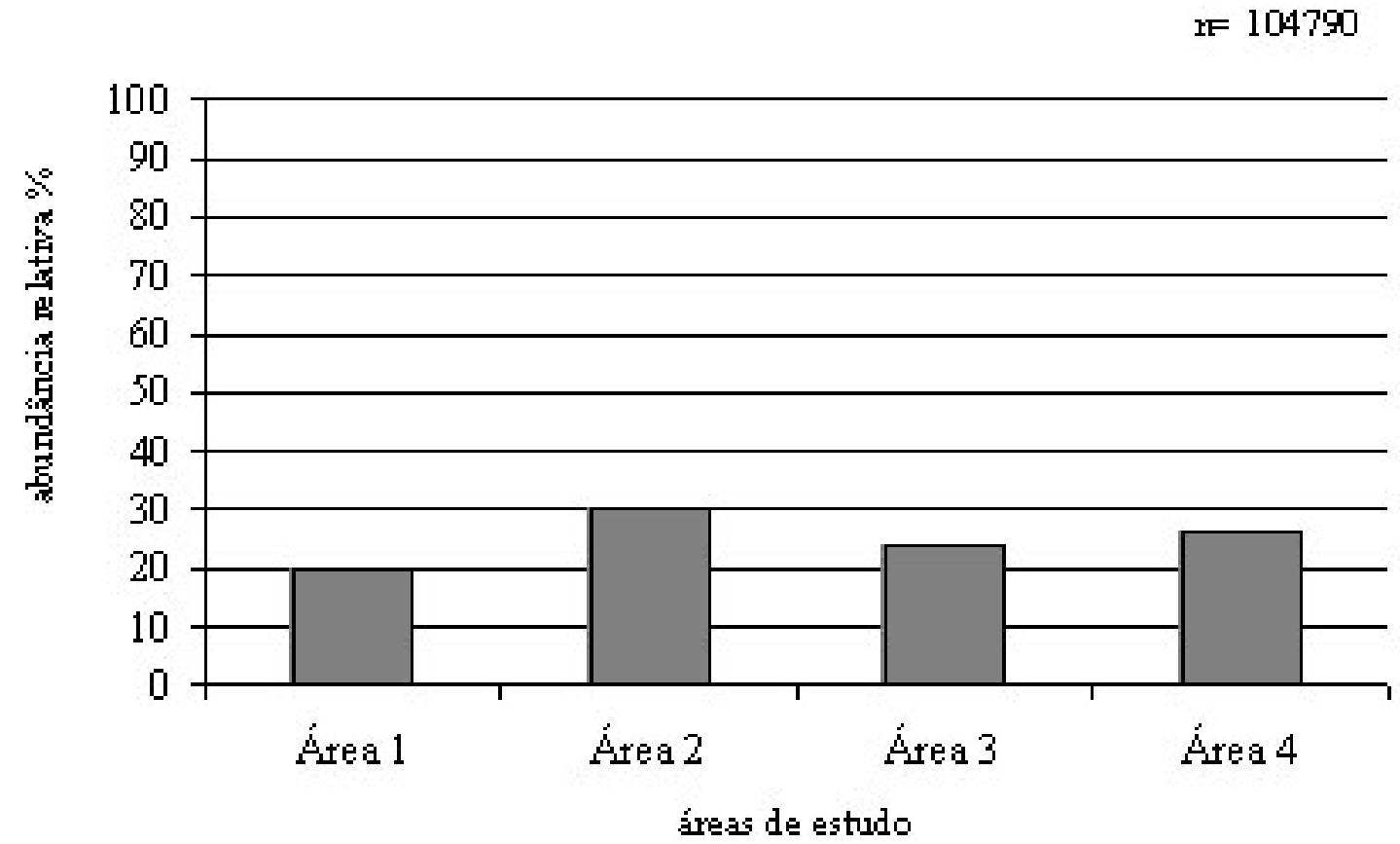

Figura 4 - Percentual de captura por área de coleta na Lagoa Rodrigo de Freitas, Rio de Janeiro. 


\section{Referências}

ANDREATA, J. V.; SAAD, A. M.; BARBIÉRI, L R. R. Associação e distribuição das espécies de peixes na Laguna de Marapendi, Rio de Janeiro, Brasil. Atlântica, Rio Grande, v. 12, n. 1, p. 5 - 17, 1989.

ANDREATA, J. V. et al. Relação dos peixes da Laguna de Marapendi, Rio de Janeiro, Brasil. Atlântica, Rio Grande, v. 12, n. 1, p. 5 - 17, 1990a.

ANDREATA, J. V. et al. Alguns aspectos da ecologia das espécies de peixes da Laguna da Tijuca, período de março de 1987 a fevereiro de 1989, Rio de Janeiro, Brasil, Acta Biológica Leopoldensia., São Leopoldo, v. 12, n. 2, p. 247 268, 1990b.

ANDREATA, J. V. et al. Associações, similaridade e abundância relativa dos peixes da Laguna de Jacarepaguá, Rio de Janeiro, Brasil. Boletim do Museu Nacional. Rio de Janeiro, n. 335, p.1-25, 1992.

ANDREATA, J. V. et al. Ictiofauna da laguna Rodrigo de Freitas, Rio de Janeiro, Brasil.. Universidade Santa Úrsula, Rio de Janeiro, 1998. 110p.

ANDREATA, J. V.; TEIXEIRA, D. E. P.; GUERON, O. C. Composição e distribuição espacial da macrofauna associada as macrófitas aquáticas na Lagoa Rodrigo de Freitas. In: JOSÉ V. ANDREATA (ed.) Lagoa Rodrigo de Freitas síntese histórica e ecológica. Rio de Janeiro: Ed. Universidade Santa Úrsula, 2001.

ANDREATA, J. V. et al. Assembléia de peixes da laguna Rodrigo de Freitas, Rio de Janeiro. Bioikos, Campinas v. 16, n. 1/2., p. 19 - 28, 2002.
AGUIARO, T.; CARAMASCHI, É. P. Icthyofauna composition of three coastal lagoons in the north of the state of Rio de Janeiro (Brazil). Arq. Biol. Tecnol. v.38, n. 8, p. 1181 - 1189, 1995.

KOBLTZ, J. L et al. Distribuição dos metais pesados nos sedimentos recentes da Laguna Rodrigo de Freitas, Rio de Janeiro, Brasil. In: ANDREATA J. V. (ed.) Lagoa Rodrigo de Freitas síntese histórica e ecológica. Rio de Janeiro: Ed. Universidade Santa Úrsula, 2001.

MARCA, A. G.; ANDREATA, J. V. Estrutura da comunidade de peixes da Lagoa Rodrigo de Freitas In: ANDREATA J. V. (ed.) Lagoa Rodrigo de Freitas síntese histórica e ecológica. Rio de Janeiro. Ed. Universidade Santa Úrsula, 2001. P. 77142

OLIVEIRA, J. A. Contribuição ao conhecimento da fauna da Lagoa Rodrigo de Freitas. Rio de Janeiro: Sudepe, 1976. 11p.

REIS, R. A.; AGUIARO, T., CARAMASCHI, É. P. Distribuição Espacial da Ictiofauna nas Lagoas Cabiúnas e Comprida. In: Francisco de Assis Esteves (ed) Ecologia das Lagoas Costeiras do Parque Nacional da Restinga de Jurubatiba e do Município de Macaé (RJ). Rio de Janeiro: Núcleo de Pesquisas Ecológicas de Macaé (NUPEM) - Universidade Federal do Rio de Janeiro, Rio de Janeiro, 1998.
Recebido em/Recived in: 04.05.2004. Aprovado em/Approved in: 31.05.2004. 